\title{
Mudanças climáticas e mitigação no setor florestal: REDD+, políticas nacionais e desenvolvimento sustentável local na Amazônia Legal ${ }^{*}$
}

\author{
Douglas Sathler ${ }^{\star *}$ \\ Susana B. Adamo ${ }^{\star \star \star}$ \\ Everton E. C. Lima ${ }^{\star \star \star \star}$
}

Existe uma mobilização global em prol de iniciativas de mitigação e adaptação às mudanças climáticas, com a intensificação das discussões sobre o tema na academia e nos fóruns internacionais. Diante disso, a preservação das florestas tropicais tem ganhado especial atenção, tendo em vista seu potencial mitigador (BUSCH; FERETTI-GALLON, 2014) e, também, as consequências regionais do desmatamento e da degradação das florestas (NOBRE et al., 2009). Nesse contexto, a REDD+ (Reducing Emissions from Deforestation and Forest Degradation and Enhancement of (arbon Stocks) tem desenvolvido parcerias para disseminar conhecimento e alavancar fundos para a promoção de iniciativas de proteção às florestas dos países em desenvolvimento (ANGELSEN, 2012; UN-REDD, 2015). A inserção do Brasil na REDD+e as políticas nacionais para a redução do desmatamento na Amazônia Legal ${ }^{1}$ têm sido largamente discutidas em fóruns domésticos e internacionais.

\footnotetext{
* Este trabalho contou com o financiamento da Coordenação de Aperfeiçoamento de Pessoal de Nível Superior (Capes), Brasil. ** Universidade Federal dos Vales de Jequitinhonha e Mucuri (UFVJM), Unaí-MG, Brasil, visiting scholar Center for International Earth Science Information Network (Ciesin), Columbia University, Palisades, NY, Estados Unidos (doug.sathler@gmail.com). ${ }^{* \star \star}$ Center for International Earth Science Information Network (Ciesin), Columbia University, Palisades, NY, Estados Unidos (sadamo@ciesin.columbia.edu).

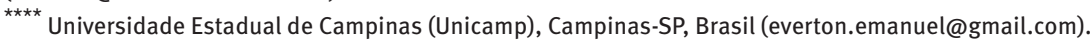

${ }^{1}$ A Amazônia Legal é formada por 771 municípios, distribuídos em nove estados (Acre, Amazonas, Rondônia, Roraima, Mato Grosso, Pará, Maranhão, Tocantins e Macapá).
} 
Na Amazônia Legal, é preciso avançar nas ações que têm demonstrado bons resultados no curto prazo, a exemplo do monitoramento e controle do desmatamento, assim como investir em iniciativas que irão estancar definitivamente o desmatamento na região (NEPSTAD et al., 2009). As contribuições da comunidade internacional e a atuação dos diferentes níveis de governo na região devem avançar na promoção do desenvolvimento sustentável local, para garantir a criação de um ciclo de preservação duradouro em sintonia com as principais demandas da população (MER et al., 2011).

Nas discussões sobre mudanças climáticas, mitigação no setor florestal e desenvolvimento sustentável local, cabe ressaltar a contribuição das ciências sociais por meio da promoção de estudos interdisciplinares que exploram as interseções entre as dinâmicas social, econômica e demográfica da Amazônia Legal. Nesse espírito, uma parceria recente entre pesquisadores da Universidade Federal dos Vales do Jequitinhonha e Mucuri (UFVJM), do Center for International Earth Science Information Network (Ciesin) da Columbia University e do Núcleo de Estudos de População (Nepo) da Universidade Estadual de Campinas (Unicamp) tem contribuído para a ampliação destas discussões.

Esta nota de pesquisa oferece, inicialmente, uma análise da atuação da REDD+e das políticas nacionais no combate ao desmatamento na Amazônia Legal. Posteriormente, o texto traz reflexões sobre a importância do desenvolvimento sustentável local para a mitigação na região e as interações entre o desmatamento e os padrões de desenvolvimento local na Amazônia Legal. Mais adiante, são feitos alguns apontamentos sobre as possíveis contribuições da demografia nos estudos sobre desmatamento e desenvolvimento sustentável local na Amazônia Legal. Ao final, apresenta-se uma breve descrição dos próximos passos da pesquisa.

\section{Desmatamento, REDD+ e políticas nacionais na Amazônia Legal}

O quinto relatório de avaliação do Painel Intergovernamental sobre Mudanças Climáticas (IPCC) revelou que o desmatamento e a degradação das florestas formam a segunda maior fonte de emissões humanas de $\mathrm{CO}^{2}$ em 2013, sendo responsáveis por cerca de $10 \%$ das emissões de gases estufa do mundo (IPCC, 2015). Estes achados justificam o crescente interesse na comunidade internacional por políticas de preservação da floresta e mitigação no setor florestal, envolvendo países desenvolvidos e em desenvolvimento (SUMIT CAKRAVARTY et al., 2012; BUSCH; FERRETI-GALLON, 2014).

Em 2007, a terceira conferência das partes (COP13) da Convenção Quadro das Nações Unidas sobre as Mudanças Climáticas (UNFCCC) amadureceu um importante instrumento para recompensar nações em desenvolvimento por suas práticas de mitigação no setor florestal: a REDD+, que atualmente tem várias iniciativas para promover a conservação, o manejo sustentável das florestas e o melhoramento dos estoques de carbono, com destaque para o UN-REDD Programme, a FCPF (Forest Carbon Partnership Facility) e o FIP (Forest Investment Program) (ANGELSEN et al., 2012). 
Nos circuitos internacionais, as potenciais contribuições do Brasil para a mitigação em escala planetária merecem atenção especial. 0 país abriga um terço das florestas tropicais do mundo, incluindo $65 \%$ da Amazônia. Diante disso, os efeitos ecológicos do desmatamento no Brasil são potencialmente grandes e o sistema climático global pode ser afetado significativamente (HOUGTON et al., 2001; SOARES-FILHO, 2006). O Brasil é considerado um parceiro-chave pela REDD+ não apenas por abrigar $413.152 \mathrm{~km}^{2}$ de florestas tropicais em seu território (INPE, 2014), mas também pela sua capacidade de financiar iniciativas e estimular cooperações Sul-Sul. De fato, o Brasil estabeleceu uma meta ambiciosa para redução do desmatamento: $80 \%$ em relação ao valor de referência (19.500 km² por ano) até 2020 (BRASIL, 2009).

Não obstante a liderança natural do Brasil nos fóruns da REDD+, o país optou por não se tornar um parceiro oficial do UN-REDD Programme e do FCPF. O Ministério do Meio Ambiente (MMA) argumenta que as estratégias de combate ao desmatamento devem ser determinadas domesticamente. Contudo, o Brasil deverá ser o primeiro país a ter seus resultados de REDD+ reconhecidos pela UNFCCC de acordo com o MMA (BRASIL, 2015).

Nesse contexto, o desmatamento e a degradação florestal na Amazônia estão no centro dos esforços brasileiros para a mitigação das mudanças climáticas. Em 2004, o país apresentou seu Plano de Prevenção e Controle do Desmatamento na Amazônia Legal (PPCDAm), concebido para guiar ações multissetoriais no combate ao desmatamento, estabelecendo prioridade e criando diálogo com outras iniciativas complementares. Este plano definiu estratégias para as seguintes áreas: monitoramento e controle ambiental; ordenamento fundiário e territorial; e fomento às atividades produtivas sustentáveis. O PPCDAm prevê iniciativas em sintonia com as metas da REDD+, com o Plano Amazônia Sustentável (PAS, 2008), com a Política Nacional sobre Mudanças do Clima (Lei n. 12.187/2009) e com o Fundo Amazônia.

Assunção et al. (2012) demonstraram que as políticas do PPCDAm têm contribuído significativamente para reduzir o ritmo do desmatamento na Amazônia Legal. Os autores estimaram que, entre 2005 e 2009 , estas políticas evitaram de $27 \%$ a $62 \%$ do desmatamento na região, o que representa de 270 a 621 toneladas de $\mathrm{CO}^{2}$ que deixaram de ser lançadas na atmosfera. 0 sucesso das políticas brasileiras de proteção da Amazônia Legal também é descrito em estudos que destacam a melhoria do monitoramento (INPE, 2014), a recente expansão das reservas indígenas e de áreas protegidas (SOARES-FILHO et al., 2010), o fortalecimento das leis para exploração madeireira (UCS, 2011) e as parcerias do setor público com organizações não governamentais (ONGs) e com o setor privado (GREEN PEACE, 2009).

De fato, o Inpe registrou, em 2014, uma taxa de desmatamento 5,54 vezes menor do que a observada em 2004. De acordo com Nepstad et al. (2009, p. 1350), as políticas internacionais e esta desaceleração das taxas "finalmente torna viável o fim do desmatamento na Amazônia Legal, o que resultaria numa redução de 2 a $5 \%$ das emissões globais de carbono". Entretanto, não existe garantia de que este será um padrão definitivo. 
O Instituto de Pesquisas Econômicas e Aplicadas (Ipea), a Sociedade Alemã para Cooperação Internacional (GIZ) e a Comissão Econômica das Nações Unidas para a América Latina e Caribe (Cepal) avaliaram recentemente os impactos do PPCDAm. Esta avaliação, disponibilizada em 2011, indicou que a maior parte das ações efetivas do PPCDAm esteve focada no controle e monitoramento do desmatamento da Amazônia Legal. Conforme este estudo, as ações que devem garantir uma redução duradoura do desmatamento (ordenamento fundiário e territorial e fomento às atividades produtivas sustentáveis) não tiveram um nível satisfatório de efetividade (IPEA; GIZ: CEPAL, 2011).

Nos anos 2000, o desmatamento da Amazônia Legal esteve muito atrelado às variações do preço da carne e da soja no mercado internacional (HARGRAVE; KIS-KATOS, 2003; MACEDO et al., 2011). Ainda, Andersen (1996), Barreto e Silva (2009) e Assunção et al. (2013) concluíram que existe uma forte relação entre a disponibilidade de crédito agrícola e as taxas de desmatamento na Amazônia Legal. Não obstante os esforços do MMA na última década, estes estudos sugerem que o ritmo do desmatamento na Amazônia Legal ainda continua perigosamente relacionado às oscilações de investimentos.

De maneira geral, as discussões sobre o desmatamento na Amazônia Legal promovidas pelo governo brasileiro nos fóruns da REDD+ e, também, com a sociedade civil avançaram nos últimos anos. Entretanto, o combate ao desmatamento na região requer a ampliação de melhorias em áreas estratégicas. Nos próximos anos, o Brasil deve avançar no ordenamento fundiário e nas políticas de gestão territorial, fortalecendo a presença das instituições públicas nos municípios da Amazônia Legal. Uma mudança definitiva nos padrões de desmatamento da região requer um conjunto de iniciativas que devem ser acompanhadas da melhoria geral dos indicadores sociais de desenvolvimento local, o que desencadearia um ciclo virtuoso de prosperidade em harmonia com os interesses preservacionistas na região.

\section{Desenvolvimento sustentável local na Amazônia Legal}

A literatura recente demonstra que a mitigação e o sucesso da REDD+ na Amazônia Legal dependem de políticas que considerem as interações multiescalares, abordagens bottom-up, ações multissetoriais e participação local. As interações multiescalares são fundamentais e as abordagens bottom-up devem ser incluídas como parte de um conjunto integrado de soluções (SANDBROOK et al., 2010; KORHONEN-KURKI et al., 2012; SATHLER, 2014). Ainda, para a otimização dos resultados, governos, setor privado e a sociedade civil devem trabalhar em harmonia com as metas estabelecidas regional e localmente. A falta de coordenação entre as partes interessadas pode prejudicar a gestão sustentável da floresta e limitar o alcance das metas estabelecidas no contexto da REDD+ pelo Brasil (GEBARA et al., 2014). É importante estimular o envolvimento das comunidades tradicionais e da sociedade civil, promovendo diálogos que reúnam todas as partes interessadas, tendo em vista as possibilidades e os desafios do que tem sido 
chamado de gerenciamento florestal comunitário (community-based forest management - CBFM) (AGRAWAL et al., 2008).

0 desenho e a implementação desses arranjos devem ser acompanhados de desenvolvimento sustentável local. Ao contrário, a proteção da floresta irá, necessariamente, exigir grandes esforços e uma quantia infindável de recursos financeiros. De acordo com Mer et al. (2011), a preservação da integridade do bioma amazônico atrelada à promoção do desenvolvimento sustentável local é um grande desafio para a estratégia global de mitigação e, também, para a manutenção do equilíbrio ecológico na região.

Estudos sugerem que o desmatamento na Amazônia irá provavelmente reduzir a precipitação e aumentar a temperatura na superfície e a extensão do período de seca na região (FINDELL et al., 2006; KILLEM; SOLÓRZANO, 2008; NOBRE et al., 2009). A destruição da Amazônia e as mudanças no ciclo hidrológico na região deverão impedir a reconstituição da floresta (SHUKLA et al., 2015). A possibilidade de profundas mudanças ecológicas locais sugere a necessidade, também, de soluções locais, que se replicadas muitas vezes poderão ter impactos ambientais na escala global.

Os municípios da Amazônia Legal não estão articulados em iniciativas e projetos que buscam o desenvolvimento sustentável local e a preservação da floresta. 0 planejamento e a formulação de projetos para o desenvolvimento sustentável na Amazônia Legal estão mais presentes na escala regional. Em 2008, o governo federal lançou o Plano Amazônia Sustentável, propondo um conjunto de diretrizes integradas para estimular o desenvolvimento da Amazônia Legal. O PAS incorporou muitas políticas estratégicas no nível regional que, potencialmente, podem impactar não apenas o PPCDAm e as metas REDD+ na região, mas também as ações de desenvolvimento sustentável no nível local. O PAS e seus possíveis desdobramentos podem auxiliar municípios no estabelecimento e no cumprimento de metas, em conformidade com suas especificidades. Mesmo que as políticas de mitigação e de desenvolvimento sustentável na Amazônia Legal tenham se disseminado muito mais por iniciativas top-down do que por ações locais, encontrar o equilíbrio certo nesta equação é fundamental.

Ademais, o desenvolvimento sustentável local vai muito além do aumento dos níveis de renda e da diminuição das desigualdades. Caso não sejam acompanhados de outros elementos a serem incorporados nas políticas públicas locais, maiores ganhos econômicos não serão capazes de reduzir o desmatamento na região e, em alguns casos, podem até estimular a destruição da floresta.

\section{Desmatamento e padrões de desenvolvimento na Amazônia Legal}

A avaliação dos processos sociais, econômicos e demográficos é fundamental para formulação e implementação de políticas públicas que busquem o desenvolvimento sustentável regional e local na Amazônia Legal. Também é bastante útil para identificação de áreas e de municípios sob risco de ampliação do desmatamento num futuro próximo. 
Existe um debate em curso a respeito da interpretação dos padrões sociais e econômicos na Amazônia Legal. Rodrigues et al. (2009) encontraram o padrão boom-and-bust ${ }^{2}$ na região ao estudar as variações no nível de desenvolvimento em 286 municípios em diferentes estágios de desmatamento. De acordo com este estudo, padrões de vida, alfabetismo e expectativa de vida aumentam com o início do desmatamento, mas declinam com a evolução do desmatamento. Celentano et al. (2012) também encontraram o padrão boom-and-bust na Amazônia Legal num estudo que envolveu 408 municípios.

Entretanto, Weinholds et al. (2014) questionam os resultados que indicam a existência desse padrão na Amazônia Legal. Após apresentarem vários exercícios empíricos, os autores destacam que os resultados de Rodrigues et al. (2009) "são artefatos espúrios da correlação espacial, devido principalmente às diferenças de tamanho e heterogeneidades entre os municípios do Amazonas e Maranhão" e ainda defendem que os resultados de Calentano et al. (2012) "também são frágeis e sensíveis a mudanças na sua especificação e amostra, sendo que o padrão encontrado desaparece com a inclusão das reservas florestais e de outros efeitos" (WEINHOLDS et al., 2014, p. 1). Finalmente, os autores citam vários estudos que sugerem que, em vez de um padrão boom-and-bust na Amazônia brasileira, na verdade, existe um padrão de crescimento contínuo no bem-estar e nos níveis locais de desenvolvimento (ver PINEDO-VASQUEZ et al., 2001; SEARS et al., 2007; MANGABEIRA, 2010).

Este debate permanece aberto. A Amazônia Legal é heterogênea e existem muitos fatores que diretamente interferem nas variáveis usadas pelos autores citados. Também, não seria plausível não considerar os efeitos negativos da depreciação dos recursos naturais na região. Para esta questão, especificamente, estudos de caso que apresentem maior controle de variáveis intervenientes podem ser mais promissores. Ademais, é importante ter em mente que os estudos citados por Weinholds et al. (2014) não objetivam especificamente avaliar a existência ou não de um padrão boom-and-bust na região.

Mesmo diante das melhorias gerais no bem-estar da população brasileira nos últimos 15 anos, a Amazônia Legal ainda apresenta níveis inaceitáveis de pobreza, desigualdade e vulnerabilidade social (CALENTANO; VEDOVETO, 2011). No arco do desmatamento, os municípios de pequeno e médio porte populacional estão muito longe dos centros de decisão, formando uma rede urbana desequilibrada e com vários tipos de restrições ao desenvolvimento regional (infraestrutura, investimentos públicos e privados, capacidade de financiamento, serviços educacionais, etc.) (SERRA; FERNANDES, 2004). Os diferentes padrões nos níveis de desenvolvimento na Amazônia Legal devem ser continuamente explorados, oferecendo suporte para políticas públicas que visam a promoção da sustentabilidade e a redução do desmatamento na região (SATHLER, 2009; GUEDES et al., 2012).

\footnotetext{
${ }^{2}$ Nos anos 1980, surgiu a hipótese de que o aumento no nível de desenvolvimento com o início das atividades de exploração de recursos naturais nos trópicos seria seguido de uma posterior queda estimulada pela depreciação dos recursos naturais. Este padrão, similar a um U invertido, ficou conhecido como boom-and-bust (MORAN, 1982; HECHT, 1983; SCHNEIDER et al., 2002; BARBIER, 2004).
} 


\section{Desmatamento e desenvolvimento local na Amazônia Legal: possíveis contribuições da demografia}

A análise e a interpretação de parâmetros demográficos têm grande potencial para diagnósticos, avaliações e desenhos de políticas para o desenvolvimento sustentável local e para a preservação da floresta. As variáveis demográficas estão intimamente entrelaçadas aos indicadores de desenvolvimento humano, sendo fundamentais para o entendimento da dinâmica social nas áreas de fronteira. No nível local temos várias demografias de diferentes grupos populacionais, reforçando a relevância de abordagens interdisciplinares que levam em conta as especificidades históricas e geográficas nesta escala de análise.

Os componentes da dinâmica demográfica se comportaram com bastante dinamismo no último século ao redor do mundo (PRB, 2004; UNITED NATIONS, 2015) e ainda existe muito para entender sobre as relações entre as variáveis populacionais e o ambiente (O’NEILL, 2008). Na Amazônia Legal, assim como em outras partes do mundo, a evolução da fronteira do desmatamento trouxe fortes mudanças nas dinâmicas social e demográfica de populações tradicionais, pequenos agricultores e habitantes das cidades.

O fortalecimento da demografia ambiental e, também, a ampliação das contribuições potenciais da demografia para os estudos sobre desmatamento e desenvolvimento sustentável local exigem o aprofundamento de iniciativas já percebidas na comunidade científica, mesmo que de maneira tímida, por demógrafos e não demógrafos (FREIRE DE MELLO; SATHLER, 2015). Entre elas, destacam-se o desenho e a implementação de plataformas inteligentes voltadas para a integração de diferentes fontes de dados e informações. Os levantamentos censitários e a possibilidade de integração de dados demográficos e socioeconômicos com informações sobre a dinâmica do desmatamento na Amazônia Legal abrem um interessante universo de possibilidades analíticas. Os dados censitários (IBGE, $1970,1980,1991,2000,2010)$ e as informações sobre o desmatamento na Amazônia Legal (Projeto Prodes, 2000-2014) estão disponíveis em plataformas gratuitas que permitem integração em escala municipal.

No entanto, dois avanços fundamentais se fazem necessários:

- apesar do seu acesso imediato e gratuito, seria interessante que os dados censitários e também de desmatamento estivessem reunidos numa mesma plataforma virtual, mais dinâmica e atrativa, de modo a despertar interesse não apenas de pesquisadores, mas também de estudantes, jornalistas e do público em geral. A geração instantânea de tabelas, gráficos e cartogramas sobre a dinâmica demográfica e social do desmatamento da Amazônia Legal seria um avanço importante, com reflexos inclusive na educação ambiental e na maior disponibilização destes dados pela mídia;

- a exemplo dos dados sobre o desmatamento, que estão disponíveis tanto para unidades administrativas (municípios, microrregiões, macrorregiões, mesorregiões, 
estados) como para unidades de conservação, os dados censitários no país deveriam ser disponibilizados em recortes espaciais mais condizentes com as questões ambientais contemporâneas (bacias hidrográficas, Unidades de Conservação, biomas, domínios morfoclimáticos, áreas de vulnerabilidade socioambiental, etc.), o que reduziria boa parte das limitações existentes e, também, a necessidade de esforços contínuos para a compatibilização de bases cartográficas.

Ademais, a demografia ambiental deve permanecer influente e ampliar sua participação não apenas nos estudos sobre o desmatamento e o desenvolvimento sustentável local, mas também em todas as grandes questões ambientais que afligem o planeta (HOGAN, 2007). De modo geral, deve-se garantir uma participação mais efetiva da demografia nos grandes relatórios sobre o meio ambiente (IPCC, UNFCCC, Word Bank, REDD+, etc.). Estes estudos servem de referência para toda a comunidade científica internacional, com a indicação de campos de pesquisa promissores (FREIRE DE MELLO; SATHLER, 2015).

As possibilidades de análise da demografia não devem se limitar aos estudos de estoque, estrutura e distribuição populacional. Existe um horizonte promissor de trabalho para demógrafos e cientistas sociais envolvidos nos estudos sobre população, espaço e ambiente e, mais especificamente, nas abordagens que vislumbram o desenvolvimento sustentável local e a mitigação nos municípios da Amazônia Legal.

\section{Algumas considerações e próximos passos}

As iniciativas da comunidade internacional, sobretudo a REDD+, devem combinar soluções práticas e de curto prazo com estratégias mais arrojadas para ampliar a mitigação às mudanças climáticas no setor florestal. Na Amazônia Legal, é fundamental encorajar a participação de todos os níveis de governo na promoção de políticas integradas de desenvolvimento sustentável local e de preservação da floresta. Os stakeholders devem entender que as preocupações ambientais em todo o mundo em torno das mudanças climáticas trazem boas oportunidades para a retomada de velhas demandas, geralmente esquecidas ou de difícil solução, nas agendas locais.

As ações locais relacionadas ao desenvolvimento sustentável e à preservação da floresta podem ser ampliadas com a criação de uma rede de conhecimento que agregue municípios da Amazônia Legal. Esta rede seria importante para o compartilhamento de expertise e de propostas, estimulando a cooperação e a atração de recursos. Se esta ideia for ambiciosa demais, a participação de mais municípios da região no ICLEI (Governos Locais pela Sustentabilidade) poderia trazer bons resultados.

Ademais, análises empíricas com a integração de dados demográficos, socioeconômicos e ambientais são fundamentais para avaliações constantes na escala local. A pesquisa em curso trará, num futuro próximo, o resultado de uma série de análises de dados censitários e de desmatamento na escala municipal, com o intuito de identificar padrões estatísticos e espaciais na Amazônia Legal. Estas análises estão concentradas nos pequenos e médios 
municípios da Amazônia Legal (não capitais e com população inferior a 140 mil habitantes) que registraram altos níveis de desmatamento entre 2001 e 2010 (superior a 200 km² no período). Serão exploradas 27 variáveis que representam as seguintes dimensões: território; desmatamento e floresta; demografia; educação; desenvolvimento humano; economia; desigualdade; pobreza; e serviços básicos. Métodos multivariados serão utilizados no tratamento estatístico, tendo em vista o potencial da análise de componentes principais e da análise de cluster para descrição da variabilidade das variáveis e identificação de agrupamentos. Ainda, espera-se que o mapeamento dos resultados com a utilização de ferramentas de geoprocessamento auxilie na descrição dos padrões espaciais destes agrupamentos. As análises empíricas e a identificação de padrões espaciais deverão, necessariamente, subsidiar reflexões sobre as implicações dos resultados encontrados para as políticas públicas.

\section{Referências}

AGRAWAL, A.; CHHATRE, A.; HARDIN, R. Changing governance of the world's forests. Science, n. 320, p. 1460-1462, 2008.

ANDERSEN, L. The causes of deforestation in the Brazilian Amazon. The Journal of Environment \& Development, v. 5, n. 3, p. 309-328, 1996.

ANGELSEN, A. et al. (Ed.). Analysing REDD+: challenges and choices. Cifor, 2012.

ASSUNÇÃO, J.; GANDOUR, C.; ROCHA, R. Deforestation slowdown in the Legal Amazon: pricesor policies? Rio de Janeiro: Climate Policy Iniciative, 2012. Disponível em: 〈http:// climatepolicyinitiative.org/publication/deforestation-slowdown-in-the-legal-amazonprices-orpolicie/〉. Acesso em: jun. 2012.

Does credit affect deforestation? Evidence from a rural credit policy in the Brazilian Amazon. Rio de Janeiro: Núcleo de Avaliação de Políticas Climáticas, 2013.

BARBIER, E. B. Agricultural expansion, resource booms and growth in Latin America: implications for long-run economic development. World Development, v. 32, n. 1, p. 137-157, 2004.

BARRETO, P.; SILVA, D. The challenges to more sustainable ranching in the Amazon. The State of the Amazon, v. 14, p. 554-556, 2009.

BRASIL. Ministério do Meio Ambiente. COP-15. Copenhague. MMA, 2009.

. Ministério do Meio Ambiente. REDD+ Brasil. 2015. Disponível em: «http://redd.mma. gov.brr. Acesso em: set. 2015.

BUSCH, J.; FERRETTI-GALLON, K. Stopping deforestation: what works and what doesn't. Center for Global Development, 29 Apr. 2014 (CGD Climate and Forest Paper Series \# 3). Disponível em: 〈http://www.cgdev.org/publication/stopping-deforestation-what-works-and-what-doesnt〉.

CALENTANO, D.; VEDOVETO, M. A Amazônia e os objetivos de desenvolvimento do milênio. ARA, 2011.

CELENTANO, D. et al. Welfare outcomes and the advance of the deforestation frontier in the Brazilian Amazon. World Development, v. 40, n. 4, p. 850-864, 2012.

FREIRE DE MELLO, L.; SATHLER, D. A Demografia Ambiental e a emergência dos estudos sobre População e Consumo. Revista Brasileira de Estudos de População, v. 32, n. 2, 2015. 
FINDELL, K. L.; KNUDSON, T. R.; MILLY, P. C. D. Weak simulated extratropical responses to complete tropical deforestation. Climate, v. 19, n. 12, p. 283-2850, 2006.

GEBARA, M. F.; FATORELLI, L.; MAY, P.; ZHANG, S. REDD+ policy networks in Brazil: constraints and opportunities for successful policy making. Ecology and Society, v. 19, n. 3, p. 53, 2014.

GREENPEACE. Slaughtering the Amazon. Amsterdam, 2009. Disponivel em: 〈http://www. greenpeace.org/international/en/publications/reports/slaughtering-the-amazon〉.

GUEDES, G.; BRONDÍZIO, E.; BARBIERI, A.; ANNE, R.; PENNA-FIRME, R.; D’ANTONA, Á. Poverty and inequality in the rural Brazilian Amazon: a multidimensional approach. Human Ecology, $v$. 40, n. 1, p. 41-57, 2012.

HARGRAVE, J.; KIS-KATOS, K. Economic causes of deforestation in the Brazilian Amazon: a panel data analysis for the 2000s. Environmental Resource Economics, n. 54, p. 471-494, 2003.

HECHT, S. B. Cattle ranching in the Eastern Amazon: environmental and social implications. The Dilemma of Amazonia Development. Colorado: Westview Press, 1983.

HOGAN, D. J. População e meio ambiente: a emergência de um novo campo de estudos. In: HOGAN, D. J. (Org.). Dinâmica populacional e mudança ambiental: cenários para o desenvolvimento brasileiro. Campinas: Nepo/Unicamp, 2007.

HOUGHTON, R. A.; LAWRENCE, K. T.; HACKLER, J.; BROWN, L. S. The spatial distribution of forest biomass in the Brazilian Amazon: a comparison of estimates. Global Change Biology, v. 7, p. 731-746, 2001.

INPE. Projeto PRODES - Divulgação da taxa consolidada do desmatamento da Amazônia Legal para período ago./2013-jul./2014. São José dos Campos, 2014. Disponível em: 〈http://www. obt.inpe.br/prodes/Prodes_Taxa2014.pdf〉.

IPEA; GIZ; CEPAL. Avaliação do Plano de Ação para Prevenção e Controle do Desmatamento na Amazônia Legal: PPCDAm 2007-2010. Brasília: Ipea; GIZ; Cepal, 2011.

IPCC. Climate change 2014: synthesis report. Contribution of Working Groups I, II and III to the Fifth Assessment Report of the Intergovernmental Panel on Climate Change [Core Writing Team, R.K. Pachauri and L.A. Meyer (Ed.)]. Geneva, Switzerland, 2015.

KILLEEN, T. J.; SOLORZANO, L. A. Conservation strategies to mitigate impacts from climate change in Amazonia. Philosophical Transactions of the Royal Society B: Biological Sciences, v. 363, n. 1.498, p. 1881-1888, 2008.

KORHONEN-KURKI, K.; BROCKHAUS, M.; DUCHELLE, A.; ATMADJA, S.; TPHAM, T. T. Multiple levels and multiple challenges for REDD+. P. 91-110. In: ANGELSEN, A.;BROCKHAUS, M.; SUDERLIN, W.; VERCHOT, L. Analysing REDD+: challenges and choises. Borgor, Indonesia: Center for International Forestry Research, 2012.

MACEDO, M. N. et al. Decoupling of deforestation and soy production in the southern Amazon during the late 2000s. Proceedings of the National Academy of Sciences, v. 109, n. 4, p. 13411346, 09 Jan. 2012.

MANGABEIRA, J. A. de C. Serviços ecossistêmicos e trajetória de capitalização agrícola: o caso de Machadinho D'Oeste-RO. Tese (PhD) - Universidade Estadual de Campinas (Unicamp), Campinas, 2010.

MER, P. et al. Ecosystem services for poverty alleviation in Amazonia. Edimburgo: Global Canopy Programme and University of Edinburgh, 2011. 
MORAN, E. F. Ecological, anthropological, and agronomic research in the Amazon basin. Latin American Research Review, v. 17, n. 1, p. 3-41, 1982.

NEPSTAD, D. et al. The end of deforestation in the Brazilian Amazon. Science, v. 326, p. 1350-51, 04 Dec. 2009.

NOBRE, P. et al. Amazon deforestation and climate change in a coupled model simulation. Journal of Climate, v. 22, n. 21, p. 5686-97, 2009.

O'NEILL, B. et al. Population and climate change: relationships, research, and responses. Institute for the Study of Society and Environment, National Center for Atmospheric Research (NCAR) e International Institute for Applied Systems Analysis (IIASA), 2008.

PINEDO-VASQUEZ, M.; ZARIN, D. J.; COFFEY, K.; PADOCH, C.; RABELO, F. Post-boom logging in Amazonia. Human Ecology, v. 29, n. 2, p. 219-239, 2001.

PRB - Population Reference Bureau. World Population Data Sheet. 2004. Disponível em: «http:// www.prb.org>.

RODRIGUES, A.; EWERS, R.; PARRY, L.; SOUZA, C.; VERÍSSIMO, A.; BALMFORD, A. Boom-andbust development patterns across the Amazon deforestation frontier. Science, v. 324, n. 1, p. 1435-1437, 2009.

RODRIGUES-FILHO, S. et al. Road development and deforestation in Amazonia, Brazil. In: McNEILL, D.; NESHEIM, I.; BROUWER, F. Land use policies for sustainable development: exploring integrated assessment approaches, Cheltenham: Edward Elgar Publishing Limited, 2012. p. 191-216.

SANDBROOK, C.; NELSON, F.; ADAMS, W.; AGRAWAL, A.; Carbon, forests and the REDD paradox. Oryx, v. 44, n. 3, p. 330-334, 2010.

SATHLER, D. As redes para além dos rios: urbanização e desequilíbrios na Amazônia Brasileira. Belo Horizonte: Cedeplar/UFMG, 2009.

Repercussões locais das mudanças climáticas globais: urbanização, governança e participação comunitária. Caminhos de Geografia, v. 15, n. 51, 2014.

SCHNEIDER, R. R. (Ed.). Sustainable Amazon: limitations and opportunities for rural development. Washington, DC: World Bank, 2012 (Collection World Bank Technical Paper n. 515).

SEARS, R. R.; PADOCH, C.; PINEDO-VASQUEZ, M. Amazon forestry transformed: integrating knowledge for smallholder timber management in Eastern Brazil. Human Ecology, v. 35, n. 6, p. 697-707, 2007.

SERRA, M. A.; Fernández, R. G. Perspectivas para o desenvolvimento da Amazônia: motivos para o otimismo e para o pessimismo. Economia e Sociedade, v. 13, n. 2, p. 107-131, 2004.

SHUKLA, J.; NOBRE, C.; SELLERS, P. Amazon deforestation and climate change. Science, v. 247, p. 1322-25, 2015.

SOARES-FILHO, B. S. et al. Modelling conservation in the Amazon basin. Nature, v. 440, n. 7083, p. 520-23, 2006.

SUMIT CHAKRAVARTY, S. K. et al. Deforestation: causes, effects and control strategies. In: AKAIS, $0, C$. (Ed.). Global perspectives on sustainable forest management. InTech, Chapters published, 2012. p. 3-28.

UCS. Brazil's success in reducing deforestation. Cambridge, MA: Union of Concerned Scientists, Feb. 2011 (Briefing, n. 8).

UNITED NATIONS. World population prospects. New York, 2015. Disponivel em: 〈http://esa. un.org/unpd/wpp/Publications/Files/Key_Findings_WPP_2015.pdf>. 
UN-REDD. UN-REDD Programme Strategic Framework 2016-20. Research document. UNREDD Programme, 2015. Disponível em: 〈http://www.unredd.net/index.php?option=com_ docman\&view=document\&alias=14096-un-redd-pb14-2015-strategic-framework \& category _ slug=session-3-strategic-and-policy-issues\&ltemid=134>. Acesso em: 15 jul. 2015.

WEINHOLD, D. et al. Sustainability in the tropics: does a boom in deforestation lead to a bust in development? Londres: Grantham Research Institute on Climate Change and the Environment, 2012.

\section{Sobre os autores}

Douglas Sathler é graduado em geografia (Instituto de Geociências, UFMG) e doutor em Demografia (Cedeplar-UFMG). Professor da Faculdade Interdisciplinar de Humanidades (FIH) e pesquisador do Núcleo de Geociências (Nugeo) da Universidade Federal dos Vales do Jequitinhonha e do Mucuri (UFVJM). Atualmente, trabalha como bolsista pós-doc da Capes no Center for International Earth Science Information Network (Ciesin, Columbia University).

Susana B. Adamo é graduada em geografia (University of Buenos Aires), mestre em população, recursos naturais e ambiente (Facultad Latinoamericana de Ciencias Sociales, FLACSO) e Ph.D em Demografia (University of Texas at Austin). Pesquisadora do Center for International Earth Science Information Network (Ciesin, Columbia University).

Everton E. C. Lima é graduado em Ciências Políticas e Sociais (Université Libre de Bruxeles), mestre em Sociologia (Université Libre de Bruxeles) e doutor em demografia (Cedeplar-UFMG). Professor da Universidade Estadual de Campinas (Unicamp) e pesquisador do Núcleo de Estudo de População (Nepo) da Unicamp.

\section{Endereço para correspondência}

\section{Douglas Sathler}

Universidade Federal dos Vales do Jequitinhonha e do Mucuri, campus JK Rodovia MGT 367, Km 583, n. 5000, Alto da Jacuba

39100-000 - Adamantina-MG, Brasil

\section{Susana B. Adamo}

Ciesin. Columbia University

61 Route 9w. PO Box 1000. Palisades

10964 - Nova lorque, Estados Unidos.

Everton E. C. Lima

Nepo/Unicamp

Cidade Universitária Zeferino Vaz - Barão Geraldo

13083-970, Campinas-SP, Brasil

Recebido para publicação em 13/10/2015

Recomendado para publicação em 25/10/2015

Aceito para publicação em 08/11/2015 